\title{
RESEARCH
}

Open Access

\section{"Benign intracranial hypertension" is a misleading name for a more serious disease: analysis of the visual outcome in cases with idiopathic intracranial hypertension}

\author{
Ali R. Hamdan ${ }^{1 *}$ (D), Amr M. Tayel ${ }^{1}$, Eslam El Sayed El Khateeb ${ }^{1}$ and Osama Elsoghiar ${ }^{2}$
}

\begin{abstract}
Objective: Assessment of visual field outcomes following the medical or surgical management of increased intracranial pressure in patients with idiopathic intracranial hypertension.

Patient and methods: Thirty-two patients diagnosed with idiopathic intracranial hypertension who met all the modified Dandy criteria were included in this study. Magnetic resonance brain imaging with contrast and magnetic resonance venography were performed to exclude the presence of secondary causes of intracranial hypertension. Following normal neuroimaging, a lumbar puncture was performed for all patients with papilledema to measure the cerebrospinal fluid opening pressure. The assessment of visual function, including visual acuity, fundus examination (dilated fundus examination to confirm the presence and the grade of papilledema and to exclude the other ocular causes of optic disc swelling), and formal visual field assessment by perimetry when visual acuity was more than 1/60 were performed by the ophthalmologist before and after medical or surgical treatment.

Results: Thirty-two patients were included, the majority of whom were females of childbearing age (29/32; 90.6\%). All patients complained of headache. Visual assessment revealed the presence of blurry vision in all patients. Papilledema with different grades was observed in 31/32 (96.9\%) patients, while the other patient (3.1\%) showed optic atrophy; 30/ 32 (93.8\%) patients had visual acuity >1/60. The average visual field was $-18 \mathrm{~dB} \pm 9.97 \mathrm{SD}$ in the right eye and -19.47 $\mathrm{dB} \pm 5.43 \mathrm{SD}$ in the left eye. Twenty-nine patients received medical treatment in the form of acetazolamide, which succeeded in controlling idiopathic intracranial hypertension in 24/29 (82.7\%) patients, while a theco-peritoneal shunt was inserted in 8/32 (25\%) patients. After management, statistically significant improvements in cerebrospinal fluid opening pressure, presenting manifestation, visual acuity, and field were noticed.
\end{abstract}

Conclusions: Permanent visual loss is the most feared complication of idiopathic intracranial hypertension; therefore, early diagnosis and multidisciplinary treatment are necessary to improve visual acuity and visual field.

Keywords: Idiopathic intracranial hypertension, Management, Visual outcome

\footnotetext{
* Correspondence: abdallahneuro2010@gmail.com;

Ali.hemdan@med.svu.edu.eg

'Department of Neurosurgery, South Valley University, Qena, Egypt

Full list of author information is available at the end of the article
}

SpringerOpen (c) The Author(s). 2019 Open Access This article is distributed under the terms of the Creative Commons Attribution 4.0 International License (http://creativecommons.org/licenses/by/4.0/), which permits unrestricted use, distribution, and reproduction in any medium, provided you give appropriate credit to the original author(s) and the source, provide a link to the Creative Commons license, and indicate if changes were made. 


\section{Introduction}

Idiopathic intracranial hypertension is a neurological disease that is characterized by elevated intracranial pressure in the absence of a recognizable cause $[1,2]$. The condition was called pseudotumor cerebri (PTC) in 1914. Subsequently, Foley suggested calling it "benign intracranial hypertension" because it appeared to have a much more benign neurological prognosis than other mass lesions or infections. However, recently, because of the significant visual morbidity associated with PTC, the use of the adjective "benign" is no longer considered appropriate terminology; instead, physicians prefer to use the term idiopathic intracranial hypertension (IIH) for such cases.

The most common presentation of patients with idiopathic intracranial hypertension is headache, visual changes, and papilledema, which, if left untreated, may lead to permanent loss of vision [3]. The poor visual outcome is usually associated with early onset of reduced vision, optic disc hemorrhages, grade 4 papilledema, and a high body mass index [4]. Magnetic resonance imaging (MRI) of the brain with intravenous contrast and magnetic resonance venography (MRV) are considered the modalities of choice to exclude an intracranial mass or venous sinus occlusion.

Females, especially obese females, are more susceptible to IIH when they are of reproductive age. IIH is rare in children $[5,6]$. The precise pathophysiology of IIH is unknown and is mainly related to a decrease in CSF drainage, a decrease in CSF absorption, and/or an increase in venous sinus pressure [7]. IIH is diagnosed by the modified Dandy criteria [8].

The aim of treatment is to preserve vision and reduce headaches. Conservative treatment includes weight loss, repeated lumbar punctures, and drugs that reduce CSF formation, such as acetazolamide, which is the mainstay of medical treatment. Surgical intervention is typically reserved for patients who fail to improve with medical treatment or present with fulminant IIH with impending visual loss $[9,10]$.

The aim of this study was to assess visual field outcomes following the medical and surgical management of increased intracranial pressure in patients with IIH.

\section{Patients and methods}

A hospital-based observational study was conducted with 32 patients admitted to the Neurosurgery Department at Qena University Hospital, South Valley University, between May 2016 and November 2018.

An assessment of visual outcome was performed by the ophthalmologist after either medical or surgical treatment of increased intracranial pressure in patients with IIH.

The inclusion of patients in this study was performed in accordance with the guidelines laid down in the
Declaration of Helsinki; the patients were informed about the aim of the study, and their written consent was obtained after approval was obtained from the University Hospital Ethics Committee.

Only patients who met all the modified Dandy criteria were included in our study:

- Signs and symptoms of elevated intracranial pressure

- Lack of localizing signs in the neurological examination with the exception of sixth nerve palsy

- No other cause of increased intracranial pressure can be detected on neuroimaging

- Opening CSF pressure is more than $25 \mathrm{~cm}$ in water, and the biochemical and cytological CSF analyses are normal

- Exclusion of other primary structural or systemic causes of elevated intracranial venous sinus pressure (e.g., hyperviscosity syndromes and right heart failure)

Patients with the presence of the following were excluded from our study:

- Other causes of increased intracranial tension

- History of oral contraceptive pills and drugs such as vitamin $\mathrm{A}$, tetracycline, minocycline, doxycycline, and sulfa drugs

- Evidence of cerebral venous thrombosis on MRI scan

- Pregnancy or refusal to give consent

The variables assessed in patients with IIH included age at diagnosis, sex, body mass index (BMI), drug intake, use of hormonal contraception for females, duration of symptoms, transient visual obscuration, cranial nerve palsy, and diminution of vision.

The ophthalmological evaluation included visual acuity, fundus examination (dilated fundus examination to confirm the presence and grade of papilledema and to exclude other ocular causes of optic disc swelling), and a formal visual field assessment by perimetry when the visual acuity is more than $1 / 60$. In the presence of threatened visual function, a regular ophthalmic examination must be performed because it will influence the timely management of the condition.

The radiological evaluation included standardized magnetic resonance brain imaging with contrast and magnetic resonance venography to exclude the presence of secondary causes of intracranial hypertension.

Lumbar puncture is used as a diagnostic and therapeutic test. Following normal neuroimaging, a lumbar puncture should be performed in all patients with papilledema to measure the CSF opening pressure. The 
lumbar puncture procedure was explained clearly to the patients to reduce their anxiety. The CSF opening pressure was measured while the patient was in a lateral decubitus position with extended legs. A CSF sample was sent for cytological and chemical analysis to ensure that the contents were normal.

Treatment options were either medical or surgical; patients without impending loss of visual (cutoff value) function were treated with acetazolamide (carbonic anhydrase inhibitor) to decrease the intracranial pressure. We started with an initial dose of acetazolamide $(250 \mathrm{mg})$ twice daily and then gradually increased the dose to $2 \mathrm{~g}$ /day until the regression of symptoms and signs or the drug side effects became intolerable to the patient. Weight loss was recommended for obese patients. Headache prophylaxis (migraine prophylaxis agents) in the presence of inadequate headache relief in patients with stable visual function was used. Optic nerve function was monitored with the evaluation of visual acuity, fundus, and visual field perimetry. Serial lumber punctures were performed to evaluate the CSF opening pressure every 2 weeks for a maximum of three times if there was insignificant improvement in the symptoms, mainly headache or the persistence of the grade of papilledema. If there was no response to conservative management with regard to lowering the elevated intracranial pressure or improving the reduced visual function, surgery was performed.

Lumboperitoneal shunt insertion was performed for patients with failed conservative treatment or those with impending visual loss.

\section{Results}

The current study included 32 patients with a mean age at presentation of 29.1 years $\pm 4.412 \mathrm{SD}$, the majority of whom were females of childbearing age (29/32; 90.6\%). The mean body mass index (BMI) was $33.2 \pm 3.912$ SD $\mathrm{kg} / \mathrm{m}^{2}$, and the mean CSF opening pressure obtained by $\mathrm{LP}$ at the time of diagnosis was $38.2 \pm 14.141 \mathrm{SD} \mathrm{cm}$ $\mathrm{H}_{2} \mathrm{O}$.

Regarding the clinical presentation, all patients complained of headache; the most common type was migraine-like headache (19/32; 59\%), followed by tension headache $(9 / 32 ; 28 \%)$.

Visual effects were recorded in different forms; blurry vision was recorded in all patients, transient visual obscuration in $25 / 32(78 \%)$ patients, and diplopia in $5 / 32$ (15.6\%) patients. Fundus examination revealed the presence of papilledema in $31 / 32$ (96.9\%), while the other patient $(3.1 \%)$ had optic atrophy. The papilledema grades were distributed as follows: 5/32 (15.6\%), 14/32 (43.7\%), $10 / 32(31.3 \%)$, and $2 / 32(6.3 \%)$ patients had grades 1,2 , 3 , and 4 , respectively.
Regarding visual acuity, 30/32 (93.8\%) patients had visual acuity $>1 / 60$, while the other two patients had marked diminution of visual acuity; one patient was < $1 /$ 60 , and the other had no light perception.

The average visual field perimetric mean deviation (PMD) using standard automated perimetry for $30 \mathrm{pa}-$ tients was $-18 \mathrm{~dB} \pm 9.97 \mathrm{SD}$ for the right eye and -19.47 $\mathrm{dB} \pm 5.43 \mathrm{SD}$ for the left eye. We could not evaluate the visual field in two patients due to the marked diminution of vision. Generalized reduction of the visual field was the most common defect observed at baseline in the study, followed by an enlarged blind spot; the defect was more or less symmetrical in both eyes in most cases.

Other clinical presentations, including tinnitus and vomiting, were reported in $13 / 32(40.6 \%)$ and $7 / 32$ (21.8\%) patients, respectively.

Out of 32 patients, 29 patients started treatment with acetazolamide, and acetazolamide succeeded in controlling IIH in 24/29 (82.7\%) patients who received it in a dose ranging between 0.75 and $2 \mathrm{~g} /$ day, with a mean dose of $1.37 \mathrm{~g} \pm 0.34 \mathrm{SD}$ and a mean duration of $3.19 \pm 1.3$ months. Theco-peritoneal shunts were inserted in $8 / 32$ (25\%) patients: three patients needed urgent surgery without medical treatment due to impending visual loss; four patients needed surgery after receiving acetazolamide due to failure of medical treatment, with a maximum dose of 2 $\mathrm{g} /$ day; and the remaining patient had surgery due to intolerance of the acetazolamide side effects. After management, statistically significant improvements in CSF opening pressure, presenting manifestation, visual acuity, and field were noticed. The mean CSF opening pressure for non-surgically treated patients was $15.8 \mathrm{cmH}_{2} \mathrm{O}$. Headache completely resolved in $27 / 32(84 \%)$ and improved in $5 / 32(16 \%)$ patients; tinnitus completely disappeared in $9 / 13$ (69\%) and partially improved in 4/13 (30.8\%) patients; visual acuity improved in $24 / 32$ (75\%) patients; and papilledema was resolved in all patients, and no papilledema recurrence was observed after complete resolution during 4 months of follow-up in either conservatively or surgically managed patients.

Regarding the visual field outcome, the mean PMD for the right eye was $-16.50 \mathrm{~dB} \pm 5.251 \mathrm{SD}$ with a mean improvement in PMD of $1.5 \mathrm{~dB}$, while the mean PMD for the left eye was $-16.10 \mathrm{~dB} \pm 3.872 \mathrm{SD}$ with a mean improvement in PMD of 3.37.

No increase in body weight was recorded during management except in one patient.

\section{Discussion}

In our study, the mean age was 29.1 years, and females accounted for $90.6 \%$ of all patients, which was similar to many previous studies that reported a high incidence in females, such as Zhoua et al. [11], Saber et al. [12], and Agarwal et al. [13], in which females represented 90\%, 
$86 \%$, and $82 \%$ of the total study populations, respectively. IIH is common in overweight persons, and the mean body mass index of most studies was more than $25 \mathrm{~kg} / \mathrm{m}^{2}$. In the current study, the mean body mass index was $33.2 \mathrm{~kg} / \mathrm{m}^{2}$, and it was $35.4 \mathrm{~kg} / \mathrm{m}^{2}, 35 \mathrm{~kg} / \mathrm{m}^{2}$, $34.8 \mathrm{~kg} / \mathrm{m}^{2}$, and $27.1 \mathrm{~kg} / \mathrm{m}^{2}$ in Patsalides et al. [14], Chagot et al. [15], Saber et al. [12], and Agarwal et al. [13], respectively.

The mean opening CSF pressure at the time of diagnosis varies; in the current study, it was 38.2 $\mathrm{cmH}_{2} \mathrm{O}$, which was similar to the result reported in Patsalides et al. [14] study, which was $37 \mathrm{cmH}_{2} \mathrm{O}$. Chan [16] reported a higher value for the opening CSF pressure of $41.4 \mathrm{cmH}_{2} \mathrm{O}$; on the other hand, Smith and Friedman [17] and Chagot et al. [15] obtained lower values, namely, $34.3 \mathrm{cmH}_{2} \mathrm{O}$ and 28.5 $\mathrm{cmH}_{2} \mathrm{O}$, respectively.

Clinical manifestations varied; in the current study, headache and visual complaints were the most common, followed by nausea, while diplopia and tinnitus were the least common manifesting complaints. In this study, headache was present in all patients; it was migraine-like in 19/32 (59\%), was tension-like in 9/32 (28\%), and could not be classified in 4/32 (13\%) patients. The headache was frontal, fronto-orbital, occipital, and parietal in $11 / 32$ (34\%), 8/32 (25\%), 7/32 (22\%), and 6/32 (19\%) patients, respectively.

Headache was the most common symptom in Agarwal et al. [13], Smith and Friedman [17], Watane and Patel [7], and Chagot et al. [15], as it was found in $89.3 \%, 84 \%$, $82.2 \%$, and $82 \%$ of patients, respectively. The headache characteristics in this study were similar to those of patients in Friedman et al. [18] because the most common headache phenotypes were migraine, accounting for $52 \%$; followed by tension-like, accounting for $22 \%$; and unclassified, accounting for $7 \%$ of their patients.

Friedman et al. [18] supposed that elevated CSF pressure might lead to persistent and chronic trigeminal activation and irritation. This trigeminal dysfunction could result in chronic dysregulation of the normal mechanisms that suppress inappropriate trigeminal nociceptive activity, leading to migraine-like headache. Friedman et al. [18] connected the intermittent course of the headache to the natural fluctuations in CSF pressure throughout the day. Eren et al. [3] reported that the differences in ventricular compliance and the duration of increased intracranial pressure could explain the clinical differences between IIH patients with or without headaches; they performed a retrospective study of 152 children with $\mathrm{IIH}$, and $14.5 \%$ of the patients had no headache.

Transient visual obscuration was noticed at the time of diagnosis of IIH in 25/32 (78\%) of patients with preserved vision, while it was identified in $70 \%, 69.1 \%$, and
$68 \%$ of the patients in Markey et al. [19], Wall [20], and Smith and Friedman [17], respectively.

Tinnitus was found in $40.6 \%(13 / 32)$ of the patients in this study, while it was found in $56 \%, 52.4 \%$, and $48.9 \%$ of the patients in Markey et al. [19], Agarwal et al. [13], and Saber et al. [12], respectively. Diplopia was present in $15.6 \%(5 / 32)$ of patients at the time of presentation in this study, which is more or less similar to the $17 \%$ reported by Agarwal et al. [13].

Visual complaints in our study contributed to decreased visual acuity, transient visual obscuration, papilledema, and visual field defects. All patients complained of decreased visual acuity, but we did not have a previous baseline for visual acuity. Two (6.3\%) patients had visual acuity less than $1 / 60$, one (3.1\%) patient had bilateral ability to count fingers, and the other had no light perception in either eye. Watane and Patel [7] and Friedman et al. [18] reported diminution of vision in $45.57 \%$ and $34 \%$ of their patients, respectively. Agarwal et al. [13] had a high proportion of patients with poor visual acuity (less than 6/18) and diminution of vision at presentation (36\% and 62\%, respectively), while the percent was $72.7 \%$ in Saber et al. [12].

In the current study, fundoscopic examination at the time of IIH diagnosis revealed bilateral post-papilledema optic atrophy in one $(3.1 \%)$ patient and bilateral papilledema in all other patients. The grades were as follows: $15.6 \%, 43.7 \%, 31.3 \%$, and $6.3 \%$ had grades $1,2,3$, and 4 , respectively.

Regarding visual field defects in the current study detected with standard automated perimetry, there were defects in all our patients. The average mean PMD for 30 patients was $-18 \mathrm{~dB} \pm 9.97$ in the right eye and $19.47 \mathrm{~dB} \pm 5.43$ in the left eye. We could not evaluate the visual field in two patients due to the marked diminution of vision. Chagot et al. [15] and Zhoua et al. [11] reported visual field defects in $87 \%$ and $86.7 \%$ of their patients, respectively. In Smith and Friedman [17], the average PMD at baseline was $-23.5 \mathrm{~dB}$, while in Pircher et al. [21], the average visual field mean deviation was $6.6 \pm 8.5 \mathrm{~dB}$ on the right side and $6.9 \pm 7.9 \mathrm{~dB}$ on the left side. In the current study, an enlarged blind spot was the most common defect observed at baseline followed by generalized constriction of the visual field, and the defect was more or less symmetrical in both eyes in most cases. In Markey et al. [19], an enlarged blind spot, loss of the nasal visual field, and generalized visual field constriction patterns were the most common defects and were found in $80 \%, 72 \%$, and $54 \%$ of the patients, respectively, while glaucoma-like arcuate field loss was the most common abnormality, followed by an isolated enlarged blind spot in Smith and Friedman [17].

In the current study, we achieved a reduction of 22.4 $\mathrm{cmH}_{2} \mathrm{O}$ in the CSF opening pressure, with a post- 
treatment mean value of $15.8 \mathrm{cmH}_{2} \mathrm{O}$ for non-surgically treated patients, which is similar to that reported in Patsalides et al. [14], in which they achieved a reduction of $22.9 \mathrm{cmH}_{2} \mathrm{O}$ in the CSF opening pressure after 3 months of treatment. The mean reduction in the CSF opening pressure is most likely related to the average pretreatment intracranial pressure, the tolerance of acetazolamide use, and other risk factors contributing to $\mathrm{IIH}$ development, such as high body mass index.

In our study, the headache completely disappeared in $100 \%$ of patients who received theco-peritoneal shunts and in $20 / 24$ (83\%) patients who were treated by medical treatment only. Julayanont et al. [22] reported headache improvement in $92 \%$ and $83 \%$ of patients in two different previous studies following theco-peritoneal shunt insertion.

In our study, visual acuity did not improve in the patient who had no light perception pre-operatively, but the other patient with the ability to count fingers had visual acuity of $1 / 60$ in the right eye, while the left eye did not improve. Other patients had a subjective sensation of improved visual acuity following normalization of increased intracranial pressure. Kalyvas et al. [23] achieved visual improvement and papilledema resolution for all patients after normalization of intracranial pressure, while the percent of patients who achieved visual improvement was only $70.3 \%$ and $55 \%$ in Scherman et al. [6] and Saber et al. [12], respectively. Chagot et al. [15] were characterized by a high rate of poor visual outcome at 6 months of follow-up, but they attributed that to a high percentage of patients with observed weight gain at 6 months. Agarwal et al. [13] concluded that reduced vision at baseline is a predictor of a poor visual outcome, while patients with preserved vision usually have a better prognosis.

We reported papilledema resolution in $100 \%$ of patients with pre-management papilledema. Saber et al. [12] and Scherman et al. [6] reported papilledema improvement in $85.8 \%$ and $71 \%$ of the patients, respectively, following IIH management. Agarwal et al. [13] reported that patients who had papilledema at the time of diagnosis usually had a good prognosis. Markey et al. [19] attributed the cause of papilledema to the transmission of elevated intracranial pressure via the CSF to the retrobulbar optic nerve sheath.

The visual field outcome is expressed by the change in the average perimetric mean deviation from baseline. All our patients had improved visual field on follow-up perimetry performed at 1.5 months following the complete resolution of papilledema, with the exception of the two patients with severely reduced visual acuity; the mean PMD for the right eye was $-16.50 \mathrm{~dB}$ with a mean improvement in PMD of $1.5 \mathrm{~dB}$, while the mean PMD for the left eye was $-16.10 \mathrm{~dB}$ with a mean improvement in
PMD of 3.37. Wall [21] reported complete or nearly complete resolution of visual field deficits in 92\% (11/ 12) of their patients, with a mean PMD improvement of $6.35 \mathrm{~dB}$ in the patients who received a theco-peritoneal shunt. Agarwal et al. [13] reported that patients with a poor visual acuity outcome also had a poor visual field outcome, as he had 48 patients with good visual acuity outcome and $10(17.2 \%)$ patients with poor visual outcome, and 32 patients with a good visual acuity outcome had a good visual field outcome, and 9 patients with a poor visual acuity outcome had a poor visual field outcome.

\section{Conclusion}

Permanent visual loss is the most feared complication of $\mathrm{IIH}$. The vision prognosis is generally good; however, the reduction in visual acuity, loss of visual field, and optic atrophy at presentation are predictors of a poor visual outcome. Early diagnosis and multidisciplinary treatment improve visual acuity and visual field.

\section{Study limitation}

This study was limited by the timing of the visual field follow-up, which was performed 1.5 months following the theco-peritoneal shunt insertion or complete resolution of papilledema for those who were managed non-surgically. Further assessment of the visual field on subsequent dates is needed. Additionally, the number of patients included in this study is still small. In addition, the study was partly retrospective.

\section{Abbreviations}

BMI: Body mass index; CSF: Cerebrospinal fluid; IH: Idiopathic intracranial hypertension; ICP: Intracranial pressure; MRI: Magnetic resonance imaging; MRV: Magnetic resonance venography; PMD: Perimetric mean deviation; PTC: Pseudotumor cerebri

\section{Acknowledgements}

We express our deepest gratitude to our colleagues in the Neurosurgery and Ophthalmology Departments, Qena University Hospital, South Valley University, Egypt. In addition, we would like to extend our gratitude to all the patients; without their cooperation, no work can be done.

\section{Authors' contributions}

All authors contributed equally to this work. All authors read and approved the final manuscript.

Funding

No funding received

Availability of data and materials

All data generated and analyzed during this study are available from the corresponding author upon request.

Ethics approval and consent to participate

All participants were informed about the aim of our study, and written informed consent was obtained after approval was received from the Ethics Committee of the Qena Faculty of Medicine, South Valley University (Ethics committee reference no. 06/18)

Consent for publication

Not applicable 


\section{Competing interests}

The authors declare that they have no competing interests.

\section{Author details}

'Department of Neurosurgery, South Valley University, Qena, Egypt. ${ }^{2}$ Department of Ophthalmology, Faculty of Medicine, South Valley University, Qena, Egypt.

Received: 30 July 2019 Accepted: 16 October 2019

Published online: 27 November 2019

\section{References}

1. Agarwal MR, Yoo JH. Optic nerve sheath fenestration for vision preservation in idiopathic intracranial hypertension. Neurosurg Focus. 2007;23(5):E7.

2. Ball AK, Clarke CE. Idiopathic intracranial hypertension. Lancet Neurol. 2006; 5:433-42.

3. Eren $Y$, Kabatas $N$, Yavasoglu N, et al. Idiopathic intracranial hypertension without headache: a case report and literature review. Agri J. 2018;30:142-5.

4. Mollan S, Davies B, Silver N, Shaw S, et al. Idiopathic intracranial hypertension: consensus guidelines on management. J NeurolNeurosurg Psychiatry. 2018:1-13.

5. Ballestero M, Teixeira T, Augusto $L$, et al. Cranial morcellation decompression for refractory idiopathic intracranial hypertension in children. Child's Nervous System J. 2018;34:1111-7.

6. Scherman D, Dmytriw A, Nguyen G, et al. Shunting, optic nerve sheath fenestration and dural venous stenting for medically refractory idiopathic intracranial hypertension: systematic review and meta-analysis. Ann Eye Sci J. 2018;3:26.

7. Watane $\mathrm{G}$ and Patel B: The Significance of arachnoid granulation in patients with idiopathic intracranial hypertension. J Comput Assist Tomogr, 42, 2018, March/April, article 2

8. Berezovsky D, Brucea B, Vasseneix C, et al. Cerebrospinal fluid total protein in idiopathic intracranial hypertension. Journal of the Neurological Sciences. 2017;381:226-9.

9. Falardeau J, Lobb B, Golden S, et al. The use of acetazolamide during pregnancy in intracranial hypertension patients. J Neuro-Ophthalmol. 2013; 33:9-12.

10. Kanagalingam $\mathrm{S}$ and Subramanian p: Update on idiopathic intracranial hypertension. Curr Treat Options Neurol, 2018, 20: 24

11. Zhoua D, Menga $R$, Zhanga $X$, et al. Intracranial hypertension induced by internal jugular vein stenosis can be resolved by stenting. European Journal of Neurology. 2018;25:365-72.

12. Saber $H$, Lewis $W$, Sadeghi $M$, et al. Stent survival and stent-adjacent stenosis rates following venous sinus stenting for idiopathic intracranial hypertension: a systematic review and meta-analysis. InterventNeurol. 2018; 7:490-500.

13. Agarwal A, Vibha D, Prasad $K$, et al. Predictors of poor visual outcome in patients with idiopathic intracranial hypertension $(I I H)$ : an ambispective cohort study. Clinical Neurology and Neurosurgery. 2017;159:13-8.

14. Patsalides A, Oliveira C, Wilcox J, et al. Venous sinus stenting lowers the intracranial pressure in patients with idiopathic intracranial hypertension. J NeurolnterventSurg. 2018:1-5.

15. Chagot C, Blonski M, Machu J, et al. Idiopathic intracranial hypertension: prognostic factors and multidisciplinary management. Journal of Obesity. 2017:1-10.

16. Chan J. Current concepts and strategies in the diagnosis and management of idiopathic intracranial hypertension in adults. J Neurol. 2017;264:1622-33.

17. Smith S, Friedman D. The idiopathic intracranial hypertension treatment trial: a review of the outcomes. Headache J. 2017 September:1303-10.

18. Friedman D, Subramanian P. Quiros p, et al: Headache in idiopathic intracranial hypertension: findings from the idiopathic intracranial hypertension treatment trial. Headache J. 2017;57:1195-205.

19. Markey K, Mollan S, Jensen R, et al. Understanding idiopathic intracranial hypertension: mechanisms, management, and future directions. Lancet Neurol. 2016;15:78-91.

20. Wall M. Update on idiopathic intracranial hypertension. NeurolClin. 2017 February;35(1):45-57

21. Pircher A, Montali M, Pircher J, et al: Perioptic cerebrospinal fluid dynamics in idiopathic intracranial hypertension. Front. Neurol, 9, 2018 June, article 506.
22. Julayanont $P$, Karukote $A$, Ruthirago D, et al. Idiopathic intracranial hypertension: ongoing clinical challenges and future prospects. Journal of Pain Research. 2016;9:87-99.

23. Kalyvas A, Hughes M, Koutsarnakis C, et al: Efficacy, complications and cost of surgical interventions for idiopathic intracranial hypertension: a systematic review of the literature. ActaNeurochir, 159, 2017, 33-49.

\section{Publisher's Note}

Springer Nature remains neutral with regard to jurisdictional claims in published maps and institutional affiliations.

\section{Submit your manuscript to a SpringerOpen ${ }^{\circ}$ journal and benefit from:}

- Convenient online submission

- Rigorous peer review

- Open access: articles freely available online

- High visibility within the field

- Retaining the copyright to your article

Submit your next manuscript at $\boldsymbol{\nabla}$ springeropen.com 